\title{
205.
}

\section{NOTE ON THE SUMMATION OF A CERTAIN FACTORIAL EXPRESSION.}

[From the Philosophical Magazine, vol. xIII. (1857), pp. 419-423.]

Mr KIRKMAN some months ago communicated to me a formula for the double summation of a factorial expression, to which formula he had been led by his researches on the partition of polygons. The formula in a slightly altered form is as follows: viz.

$$
\Sigma_{x} \Sigma_{y} \frac{[x+y+2]^{y}}{[y+1]^{y+1}} \frac{[x]^{y}}{[y]^{y}} \frac{[r+k-x-y]^{k-1-y}}{[k-y]^{k-y}} \frac{[r-1-x]^{k-1-y}}{[k-1-y]^{k-1-y}}=\frac{2 k}{r+3} \frac{[r+k+2]^{k}}{[k+1]^{k+1}} \frac{[r]^{k}}{[k]^{k}},
$$

the summation extending from $x=0$ to $x=r-1$, and $y=0$ to $y=k-1$. In the particular case when $k=r$, then all the terms of the series except those in which $y=x$ vanish; and putting therefore $k=r$ and $y=x$, and making a slight change in the form of the right-hand side, the formula becomes

$$
\Sigma \frac{[2 x+2]^{x}}{[x+1]^{x+1}} \frac{[2 r-2 x]^{r-1-x}}{[r-x]^{r-x}}=4 \frac{[2 r+1]^{r-2}}{[r-1]^{r-1}},
$$

the summation extending from $x=0$ to $x=r-1$.

We have, in the notation of Gauss, $[m]^{m}=m(m-1) \ldots 2.1=\Pi m$, and a factorial $[m]^{n}$ is expressed in terms of the function $\Pi$ by the formula $[m]^{n}=\Pi m \div \Pi(m-n)$. Write also

$$
\Pi_{1}\left(m-\frac{1}{2}\right)=\left(m-\frac{1}{2}\right)\left(m-\frac{3}{2}\right) \ldots \frac{3}{2} \cdot \frac{1}{2},
$$

we have

$$
\begin{aligned}
& \Pi 2 m=2^{2 m} \quad \Pi m \Pi_{1}\left(m-\frac{1}{2}\right), \\
& \Pi(2 m+1)=2^{2 m+1} \Pi m \Pi_{1}\left(m+\frac{1}{2}\right) ;
\end{aligned}
$$


and transforming the factorials by these formulæ, the series becomes

$$
\Sigma \frac{\Pi_{1}\left(x+\frac{1}{2}\right) \Pi_{1}\left(r-x-\frac{1}{2}\right)}{\Pi(x+2) \Pi(r-x+1)}=\frac{2 r \Pi_{1}\left(r+\frac{1}{2}\right)}{\Pi(r+3)},
$$

the summation, as before, from $x=0$ to $x=r-1$. This may be written

$$
\Sigma \frac{\Pi_{1}\left(x+\frac{1}{2}\right)}{\Pi_{1}\left(\frac{1}{2}\right)} \cdot \frac{\Pi_{1}\left(r-x-\frac{1}{2}\right)}{\Pi_{1}\left(r-\frac{1}{2}\right)} \frac{\Pi 2}{\Pi(x+2)} \frac{\Pi(r+1)}{\Pi(r-x+1)}=\frac{8 r\left(r+\frac{1}{2}\right)}{(r+2)(r+3)},
$$

the summation from $x=0$ to $x=r-1$. The general term does not vanish for $x=r$ or $x=r+1$, but it vanishes for all greater values of $x$; hence if we add to the right-hand side the two terms corresponding to $x=r$ and $x=r+1$, the summation may be extended from $x=0$ to $x=r+1$, or what is the same thing, from $x=0$ indefinitely. The two terms in question are

$$
\frac{4\left(r+\frac{1}{2}\right)}{r+2}+\frac{-8\left(r+\frac{3}{2}\right)\left(r+\frac{1}{2}\right)}{(r+2)(r+3)},=\frac{-4 r\left(r+\frac{1}{2}\right)}{(r+2)(r+3)}
$$

and the resulting equation is

$$
\Sigma \frac{\Pi_{1}\left(x+\frac{1}{2}\right)}{\Pi_{1}\left(\frac{1}{2}\right)} \frac{\Pi\left(r-x-\frac{1}{2}\right)}{\Pi_{1}\left(r-\frac{1}{2}\right)} \frac{\Pi 2}{\Pi(x+2)} \frac{\Pi(r+1)}{\Pi(r-x+1)}=\frac{4 r\left(r+\frac{1}{2}\right)}{(r+2)(r+3)},
$$

the summation from $x=0$ indefinitely; or substituting for the functions $\Pi$ and $\Pi_{1}$ their values, the formula is

$$
1+\frac{\frac{3}{2} \cdot(r+1)}{3 \cdot\left(r-\frac{1}{2}\right)}+\frac{\frac{3}{2} \cdot \frac{5}{2} \cdot(r+1) r}{3 \cdot 4 \cdot\left(r-\frac{1}{2}\right)\left(r-\frac{3}{2}\right)}+\frac{\frac{3}{2} \cdot \frac{5}{2} \cdot \frac{7}{2} \cdot(r+1) r(r-1)}{3 \cdot 4 \cdot 5 \cdot\left(r-\frac{1}{2}\right)\left(r-\frac{3}{2}\right)\left(r-\frac{5}{2}\right)}+\& c .=\frac{4 r\left(r+\frac{1}{2}\right)}{(r+2)(r+3)},
$$

which is a formula obviously belonging to the theory of the hypergeometric series.

$$
\mathrm{F}(\alpha, \beta, \gamma, x)=1+\frac{\alpha \cdot \beta}{1 \cdot \gamma} x+\frac{\alpha \cdot \alpha+1 \cdot \beta \cdot \beta+1}{1 \cdot 2 \cdot \gamma \cdot \gamma+1} x^{2}+\& c . ;
$$

but the formula applicable to the case in hand has probably not been given. It may be proved as follows, premising that I disregard all difficulties arising from infinite values of the functions in the definite integrals, convergency, \&c. We have

$$
\begin{aligned}
& \int_{0}^{1} \theta^{\alpha-1}(1-\theta)^{-\alpha-\gamma-1}(1-x \theta)^{\beta} d \theta \\
& \quad=\frac{\Pi(\alpha-1) \Pi(-\alpha-\gamma-1)}{\Pi(-\gamma-1)}\left(1+\frac{\alpha \cdot \beta}{1 \cdot \gamma} x+\frac{\alpha \cdot \alpha+1 \cdot \beta \cdot \beta-1}{1 \cdot 2 \cdot \gamma \cdot \gamma-1} x^{2}+\ldots\right) .
\end{aligned}
$$

Now we have

$$
\int_{0} d x \int_{0} d x(1-x \theta)^{\beta}=\frac{1}{(\beta+1)(\beta+2) \theta^{2}}(1-x \theta)^{\beta+2}-\frac{1}{(\beta+1)(\beta+2)} \frac{1}{\theta^{2}}+\frac{x}{(\beta+1) \theta}
$$


and hence multiplying by $d x$ and integrating from $x=0$, and again multiplying by $d x$ and integrating from $x=0$ to $x=1$, we find

$$
\begin{gathered}
\int_{0}^{1} d \theta \cdot \theta^{a-3}(1-\theta)^{-\alpha-\gamma+\beta+1}-\int_{0}^{1} d \theta \cdot \theta^{\alpha-3}(1-\theta)^{-a-\gamma-1}+(\beta+2) \int_{0}^{1} d \theta \cdot \theta^{\alpha-2}(1-\theta)^{-\alpha-\gamma-1} \\
=\frac{(\beta+1)(\beta+2) \Pi(\alpha-1) \Pi(-\alpha-\gamma-1)}{\Pi(-\gamma-1)} \frac{1}{2} S,
\end{gathered}
$$

if for shortness

$$
S=1+\frac{\alpha \cdot \beta}{3 \cdot \gamma}+\frac{\alpha \cdot \alpha+1 \cdot \beta \cdot \beta-1}{3 \cdot 4 \cdot \gamma \cdot \gamma-1}+\& c
$$

Substituting for the definite integrals their values,

$$
\begin{gathered}
\frac{\Pi(\alpha-3) \Pi(-\alpha-\gamma+\beta+1)}{\Pi(-\gamma+\beta-1)}-\frac{\Pi(\alpha-3) \Pi(-\alpha-\gamma-1)}{\Pi(-\gamma-3)}+(\beta+2) \frac{\Pi(\alpha-2) \Pi(-\alpha-\gamma-1)}{\Pi(-\gamma-2)} \\
=\frac{(\beta+1)(\beta+2) \Pi(\alpha-1) \Pi(-\alpha-\gamma-1)}{\Pi(-\gamma-1)} \frac{1}{2} S,
\end{gathered}
$$

whence

$$
\begin{aligned}
\frac{1}{2}(\beta+1)(\beta+2) S= & \frac{\Pi(\alpha-3)}{\Pi(\alpha-1)} \frac{\Pi(-\alpha-\gamma+\beta+1)}{\Pi(-\alpha-\gamma-1)} \frac{\Pi(-\gamma-1)}{\Pi(-\gamma+\beta-1)} \\
& -\frac{\Pi(\alpha-3)}{\Pi(\alpha-1)} \cdot \frac{\Pi(-\gamma-1)}{\Pi(-\gamma-3)}+(\beta+2) \frac{\Pi(\alpha-2)}{\Pi(\alpha-1)} \cdot \frac{\Pi(-\gamma-1)}{\Pi(-\gamma-2)}
\end{aligned}
$$

The second and third terms are

$$
-\frac{1}{(\alpha-1)(\alpha-2)}(\gamma+1)(\gamma+2)-(\beta+2) \frac{1}{\alpha-1}(\gamma+1)
$$

which are

$$
=-\frac{\gamma+1}{(\alpha-1)(\alpha-2)}(\alpha \beta+2 \alpha-2 \beta+\gamma-2) \text {. }
$$

For the reduction of the first term we have

$$
\begin{aligned}
& \Pi(-\alpha-\gamma+\beta+1)=[\beta+1-\alpha-\gamma]^{\beta+2} \Pi(-\alpha-\gamma-1) \\
& \Pi(\beta-\gamma-1) \quad=[\beta-\gamma-1]^{\beta} \Pi(-\gamma-1) ;
\end{aligned}
$$

and we thus find

$$
\frac{1}{2}(\beta+1)(\beta+2)(\alpha-1)(\alpha-2) S=\frac{[\beta+1-\alpha-\gamma]^{\beta+2}}{[\beta-\gamma-1]^{\beta}}-(\gamma+1)(\alpha \beta+2 \alpha-2 \beta+\gamma-2),
$$

where, as before,

$$
S=1+\frac{\alpha \cdot \beta}{3 \cdot \gamma}+\frac{\alpha \cdot \alpha+1 \cdot \beta \cdot \beta-1}{3 \cdot 4 \cdot \gamma \cdot \gamma-1}+\& c .
$$


205] NOTE ON THE SUMMATION OF A CERTAIN FACTORIAL EXPRESSION. 253 this is the formula in hypergeometric series required for the present purpose, and it is certainly true when the series is finite.

Write now

$$
\alpha=\frac{3}{2}, \quad \beta=r+1, \quad \gamma=r-\frac{1}{2} ;
$$

then the first term is $[1]^{r+2} \div\left[\frac{1}{2}\right]^{r+1}$, which vanishes on account of the numerator, and the second term is $-\frac{1}{2} r\left(r+\frac{1}{2}\right)$, and we have consequently

$$
-\frac{1}{2} r(r+2)(r+3) \frac{1}{2} \cdot \frac{1}{2} S=-\frac{1}{2} r\left(r+\frac{1}{2}\right)
$$

which gives

$$
S=\frac{4 r\left(r+\frac{1}{2}\right)}{(r+2)(r+3)}
$$

$S$ being here the series in $r$, the sum of which was required, and the particular case of Mr Kirkman's formula is thus verified. It is probable that the general case might be treated in an analogous manner by first grouping together the terms which correspond to a given difference $x \sim y$, and ultimately summing the sums of these partial series; but I have not examined this question.

2, Stone Buildings, W.C., April 18, 1857. 